\title{
Expression, clinical significance and mechanism of Slit 2 in papillary thyroid cancer
}

\author{
RONG-LIANG SHI ${ }^{1,2^{*}}$, NING QU $^{1 *}$, TIAN LIAO $^{1}$, YU-LONG WANG $^{1}$, \\ YU WANG ${ }^{1}$, GUO-HUA SUN ${ }^{1}$ and QING-HAI JI ${ }^{1}$ \\ ${ }^{1}$ Department of Head and Neck Surgery, Fudan University Shanghai Cancer Center; Department of Oncology, \\ Shanghai Medical College, Fudan University, Shanghai 200032; ${ }^{2}$ Department of General Surgery, \\ Minhang Hospital, Fudan University, Shanghai 201199, P.R. China
}

Received January 15, 2016; Accepted February 11, 2016

DOI: $10.3892 /$ ijo.2016.3412

\begin{abstract}
Thyroid cancer is a common endocrine malignancy. The last decade has seen exciting progress in understanding thyroid cancer molecular pathogenesis. Several major signaling pathways and related molecular derangements have been elucidated, which represent novel diagnostic and prognostic molecular markers for thyroid cancer. Based on the molecular biology of thyroid cancer, a series of therapeutic targets have been developed, which provide unprecedented opportunities. Thus, histological characterization of subgroups of patients and the correct molecular characterization of patients are thought to be key aspects for future clinical management of these patients. In the present study, we identified Slit2 as a prognostic marker for thyroid cancer oncogenesis and recurrence. Mechanistically, Slit2 regulated Warburg effect in thyroid cancer cells through regulation of HIF1 $\alpha$ and HIF1 $\alpha$ transcriptional activity. Taken together, our present data uncovered Slit2 as a novel predictive marker for thyroid cancer. The mechanism study indicated that Slit 2 regulated the Warburg effect. Additional study on the function of Slit2 in thyroid cancer is required to provide new insights into the potential mechanisms of oncogenesis and recurrence potential of thyroid cancer.
\end{abstract}

\section{Introduction}

Thyroid carcinoma is an endocrine-related malignancy. Among thyroid carcinoma, papillary thyroid cancer (PTC)

Correspondence to: Professor Guo-Hua Sun or Professor Qing-Hai Ji, Department of Head and Neck Surgery, Fudan University Shanghai Cancer Center; Department of Oncology, Shanghai Medical College, Fudan University, Shanghai 200032, P.R. China

E-mail: sunghayang@163.com

E-mail: jiqinghai@shca.org.cn

\section{${ }^{*}$ Contributed equally}

Key words: Slit2, papillary thyroid cancer, mechanism, prediction, management is the most common type and account for $80-90 \%$ of total thyroid carcinoma cases $(1,2)$. Although the clinical prognosis for the majority of cases is satisfactory, $14 \%$ demonstrate early recurrence and some present severe invasion, multiple lymph node metastasis and distant metastasis (3-5). Currently the progress in identification of biological markers that are useful for the diagnosis and prognosis analysis of PTC is slow (6). Thus, the correct molecular characterization and identification of patients with thyroid cancer is thought to be a key aspect for future study.

Slit refers to a family of related genes which encode a corresponding set of secreted proteins, also collectively referred to as Slit (7). The classical function of Slit in proteins is to act as midline repellents, preventing the crossing of longitudinal axons through the midline of the central nervous system of most bilaterian animal species. It also prevents the recrossing of commissural axons (8). Its canonical receptor is ROBO, and Slit/ROBO signaling is important in pioneer axon guidance (9-11). In recent years, the role of Slit family proteins in cancer has received much attention due to their role in controlling cell migration, abnormalities or absence in the expression in Slit proteins are associated with a variety of cancers (12-14). Our previous study identified Slit2 as a diagnosis and prognosis marker in gastric cancer. Mechanistically, it participates in gastric cancer oncogenesis and metastasis through regulating the AKT/ $\beta$-catenin pathway $(15,16)$.

To the best of our knowledge, the expression pattern of Slit2 and its correlation with clinicpathological parameters of PTC has not been previously reported. In the present study, we examined Slit2 expression in transcriptional and protein level, and then analyzed the correlation between Slit 2 expression and the clinicopatholocical characteristics of PTC. Moreover, we sought to elucidate the role of Slit2 in PTC through its association with glucose metabolism, which is considered to influence life and death decisions, because it provides cancer cells with building blocks for macromolecule synthesis and energy supply.

\section{Materials and methods}

Immunohistochemistry (IHC). Thyroid cancer tissues, confirmed by pathological diagnosis, were obtained from 196 
patients who underwent thyroidectomy between 2003 and 2012 at the Department of Head and Neck Surgery, Fudan University Shanghai Cancer Center. The corresponding non-tumor thyroid tissues were obtained at least $1 \mathrm{~cm}$ away from the tumor. All tissue samples were formalin-fixed and paraffin-embedded. TNM staging was classified based on the criteria of the American Joint Committee on Cancer (AJCC, 7 th edition) for thyroid cancer. The study was approved by the Human Ethics Committee/Institutional Review Board of Fudan University Shanghai Cancer Center. Written informed consent was obtained from all 196 patients. IHC staining was performed by using a highly sensitive streptavidin-biotinperoxidase detection system with thyroid cancer tissue microarrays. Rabbit polyclonal anti-Slit-2 (working dilution 1:50) was purchased from Proteintech Group Inc. (Chicago, IL, USA) and rabbit anti-HIF-1- $\alpha$ (working dilution 1:50) was purchased from Abcam (Cambridge, MA, USA). Immunolabeling was conducted using Dako EnVision + Rabbit Polymer (cat. no. K4003) from Dako (Carpinteria, CA, USA). The slides were counterstained with hematoxylin and coverslipped.

IHC scoring. The immunohistochemically stained tissue sections were scored separately by two pathologists blinded to the clinical parameters. The staining intensity was scored as 0 (negative), 1 (weak), 2 (medium) or 3 (strong). Extent of staining was scored as $(0,<5 \% ; 1,5-25 \% ; 2,26-50 \% ; 3,51-75 \%$; and $4,>75 \%$ ) according to the percentages of the positive staining areas in relation to the whole carcinoma area. Scores for staining intensity and percentage positivity of cells were then multiplied to generate the immunoreactivity score (IS) for each case. Tissues having a final staining score of $<4,4,6$ and $\geq 8$ were considered to be,,-+++ and +++ , respectively.

Total RNA extraction, reverse transcription and quantitative real-time PCR. Thyroid cancer tissues, confirmed by pathological diagnosis, were obtained from 130 patients who underwent thyroidectomy between 2012 and 2015 at the Department of Head and Neck Surgery, Fudan University Shanghai Cancer Center. Total RNA was extracted from tissues using TRIzol reagent (Invitrogen) according to the manufacturer's instructions. A total of $1 \mu \mathrm{g}$ RNA was reversetranscribed using a PrimeScript RT reagent kit (Takara Bio, Dalian, China). For quantitative real-time PCR (qPCR), cDNA was amplified using SYBR-Green Premix Ex Taq (Takara Bio) following the manufacturer's instructions. Slit-2 expression was normalized against $\beta$-actin mRNA expression in three independent experiments. The primers used for amplifying Slit2 were 5'-AACTGCCTTCGGGTAGATGC-3' (forward) and 5'-GAATGGCCCGAAGAGGTGAA-3' (reverse). The primers used for amplifying $\beta$-actin were 5'-CTACGTCGCCCTGGACTTCGAGC-3' (forward) and 5'-GATGGAGCCGCCGATCCACACGG-3' (reverse). The results were analyzed and calculated relative to cycle threshold values and then converted into fold changes.

Cell culture. PTC cell line K1, was purchased from the Institute of Biochemistry and Cell Biology at the Chinese Academy of Sciences (Shanghai, China). The cells were cultured in RPMI-1640 supplemented with 10\% fetal bovine serum, $2 \%$ streptomycin/penicillin and $1 \%$ amphotericin $\mathrm{B}$. K1 cells were cultured as monolayer in $5 \% \mathrm{CO}_{2}$ at $37^{\circ} \mathrm{C}$.

Plasmids. pLKO.1 TRC cloning vector (Addgene plasmid 10878) was used to generate shRNA-expression constructs. Targets (21 bp) against Slit2 were CCTCACCTTAATT CTTAGTTA and CCTGGAGCTTTCTCACCATAT, respectively. Scramble RNA (Addgene plasmid 1864) was used as control vector. HRE-luciferase construct (Addgene plasmid 26731), containing three hypoxia response elements from Pgk-1 gene, was used to assess HIF1 $\alpha$ transcriptional activity.

HRE luciferase assay. HIF1 $\alpha$ transcriptional activity was evaluated by using by using the Promega Dual-Luciferase ${ }^{\circledR}$ reporter $\left(D_{L} R^{\mathrm{TM}}\right)$ assay system. pRL-TK plasmid was used as control vector for expression of Renilla luciferase.

Cell proliferation analysis. Cell proliferation was examined by using Cell Counting kit-8 (CCK-8; Dojindo Molecular Technologies). In brief, cells were seeded in 96-well plates ( $3 \times 10^{3}$ cells/well), $10 \mu \mathrm{l}$ CCK-8 solution were added to each well at $0,24,48$ and $72 \mathrm{~h}$, and the plates were incubated at $37^{\circ} \mathrm{C}$ in $5 \% \mathrm{CO}_{2}$ for $1 \mathrm{~h}$. The absorbance of each sample was measured at a wavelength of $450 \mathrm{~nm}$ using a microplate reader.

Colony formation assay. K1 cells were digested and 200 cells were seeded and incubated in a fresh 6-well plate for 14 days to allow colonies to form. Colonies were fixed in methanol, stained with $0.1 \%$ crystal violet solution and counted.

Glycolysis analysis. Glucose uptake colorimetric assay kit (BioVision Inc., Milpitas, CA, USA) and Lactate colorimetric assay kit (BioVision) were purchased to examine the glycolysis process in $\mathrm{K} 1$ cells according to the manufacturer's protocol. To test expression of glycolytic enzymes and HIF1 $\alpha$ transcriptional activity, real-time PCR was performed. In brief, total RNA was prepared using TRIzol reagent (Invitrogen, Carlsbad, CA, USA), followed by reverse transcription using Takara PrimeScript RT reagent to obtain cDNA. The expression status was assessed by quantitative real-time PCR (qRT-PCR) using ABI 7900HT Real-Time PCR system (Applied Biosystems, Foster City, CA, USA). All reactions were run in triplicate. Primer sets Glut1 F, 5'-CAGTTTGGCTACAACACTGG AG-3' and R, 5'-GCCCCCAACAGAAAAGATGG-3'; HK2: F, 5'-CAAAGTGACAGTGGGTGTGG-3' and R, 5'-GCCAG GTCCTTCACTGTCTC-3'; LDHA F, 5'-CCCAGTTTCCAC CATGATTAAGG-3' and R, 5'-TTCTGTCCCAAAATGCAA GGAA-3'; PKM2 F, 5'-CAAAGGACCTCAGCAGCCATG TC-3' and R, 5'-GGGAAGCTGGGCCAATGGTACAGA-3'; $\beta$-actin F, 5'-AGAGCTACGAGCTGCCTGAC-3' and R, 5'-AGCACTGTGTTGGCGTACAG-3'.

Western blot analysis and antibodies. Protein extracts were prepared and resolved on 10\% SDS-PAGE gels, transferred to nitrocellulose membranes $(0.45 \mathrm{~mm})$ and immunoblotted with primary antibodies. Slit2, c-myc and HIF1 $\alpha$ antibodies were purchased from Abcam. $\beta$-actin antibody was used as loading control antibody. Images were developed with ECL (GE Healthcare, Pittsburgh, PA, USA). 

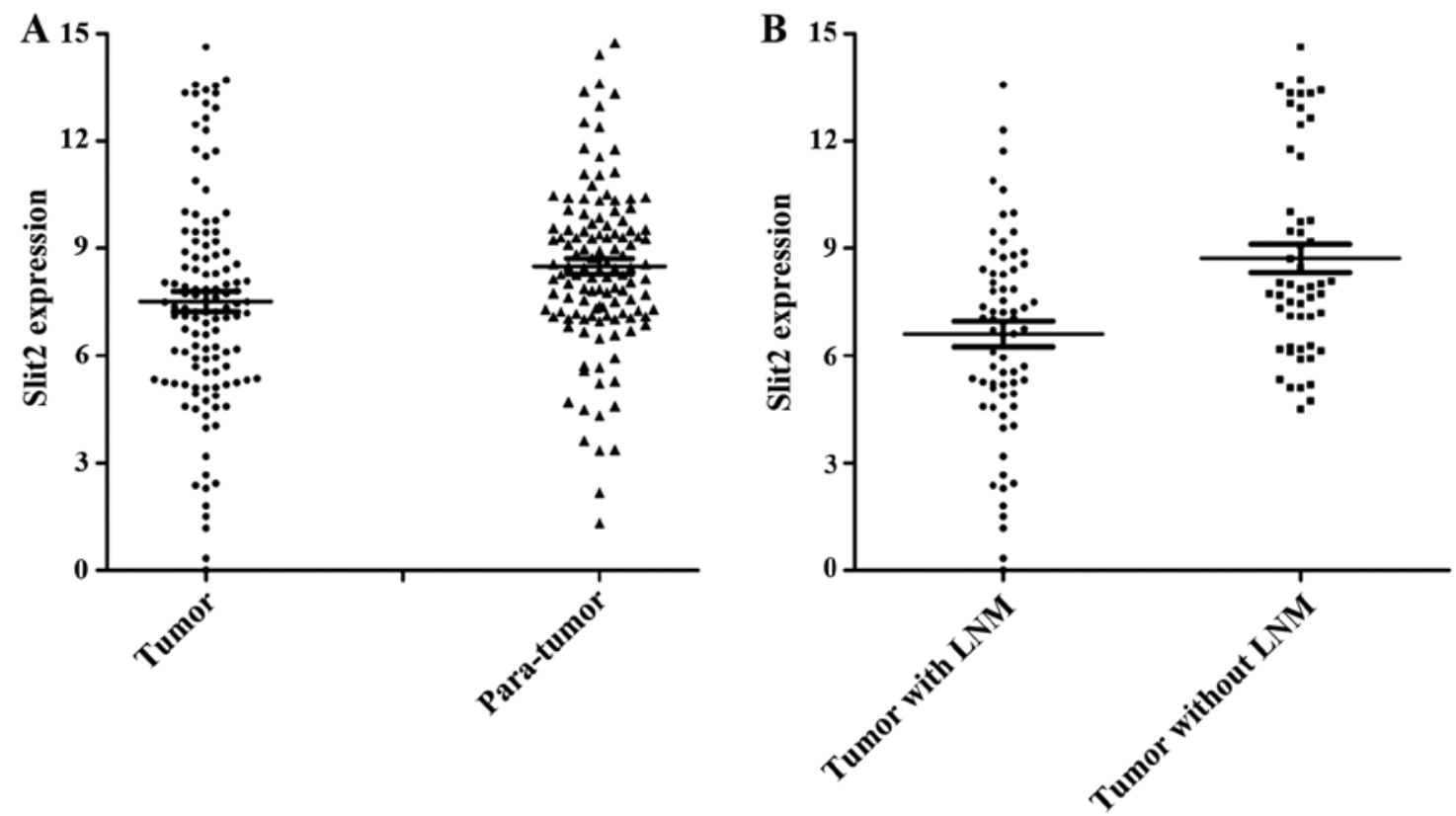

Figure 1. Slit2 mRNA expression in PTC (A) mRNA expression levels of Slit2 in PTC (tumor) and adjacent non-tumor (non-tumor) tissues were examined by qRT-PCR. Data represent the mean of triplicate experiments normalized to $\beta$-actin expression. Slit2 expression levels were decreased in PTC tumor samples. (B) Slit2 mRNA level in PTC tumors with and without LNM. Decreased Slit2 expression was associated with LNM. LNM, lymph node metastasis.
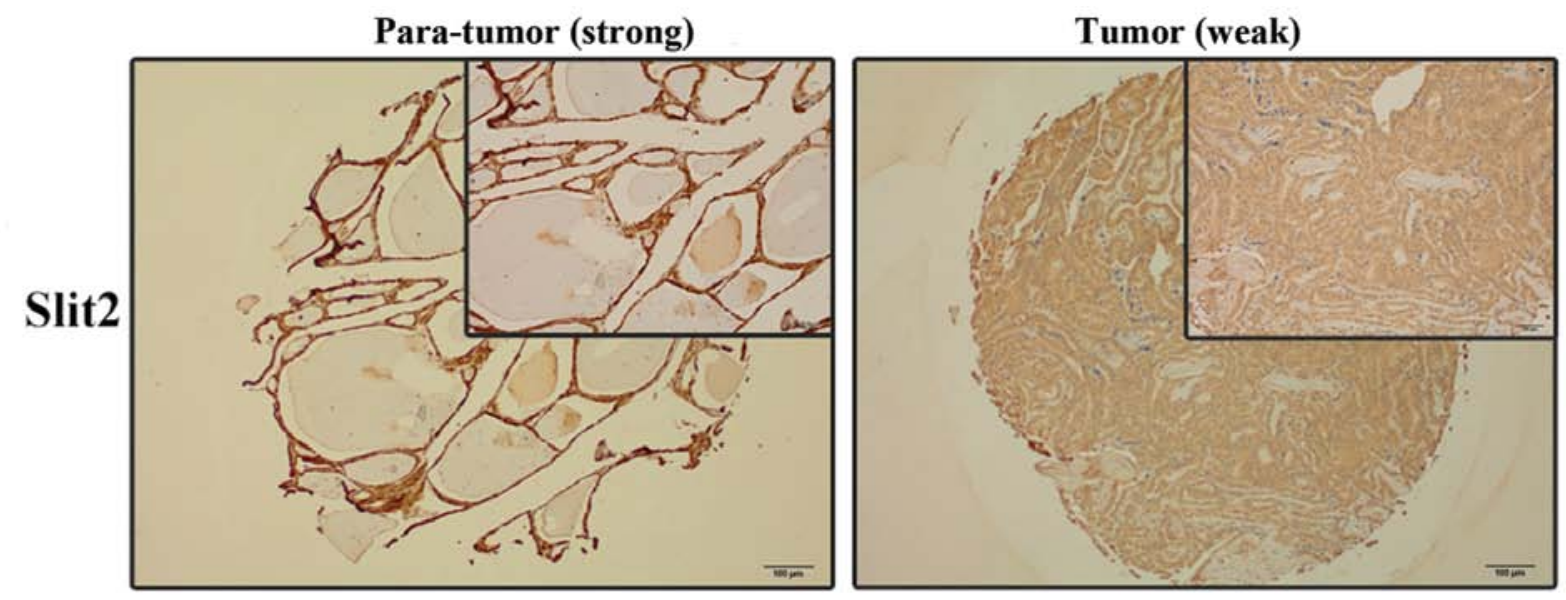

Figure 2. Slit2 protein staining indices by IHC immunohistochemical staining for Slit2 expression in thyroid cancer and adjacent normal tissues. Slit2 was expressed predominantly in the nuclei and plasma of cells in the tumor and para-tumor regions. Slit2 expression was strongly positive in para-tumor tissues and weakly positive in thyroid cancer regions.

Statistical analysis. All statistical analyses were performed using SPSS 16.0 software. Results were expressed as means \pm standard deviation. Two-tailed unpaired Student's t-tests and one-way analysis of variance were used to evaluate the data. P-values $<0.05$ were considered as statistically significant.

\section{Results}

Slit2 mRNA expression in PTC. One hundred pairs of specimens were investigated by qPCR to investigate the expression of Slit2. The average mRNA expression levels of Slit2 were significantly lower in PTC tissues compared with adjacent non-tumor thyroid tissues (Fig. 1A). Next, we examined Slit2
mRNA expression in PTC samples with lymph node metastasis (LNM). The results demonstrated that the lower Slit2 mRNA levels were associated with lymph node metastasis at diagnosis (Fig. 1B).

Slit2 protein staining indices by IHC. The protein levels of Slit2 in PTC samples were analyzed by IHC staining. Slit2 level was lower in PTC samples than that in non-tumorous samples (Fig. 2). Furthermore, to test the role of Slit2 in LNM of PTC, we examined the level of Slit2 in PTC tissue microarray containing 196 patients samples. The clinicopathological features of the patients are listed (Table I). In addition, further analysis demonstrated that Slit2 is a negative indicator for LNM at diagnosis (Table II). 
A

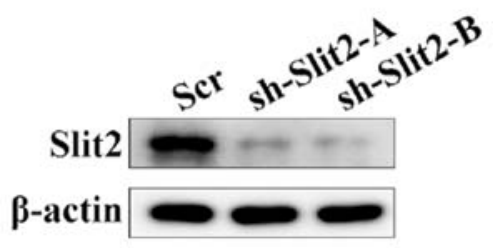

C

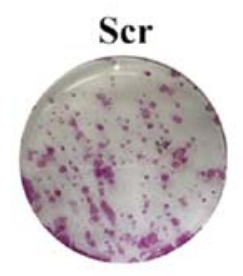

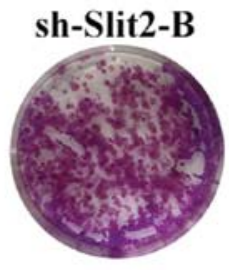

B

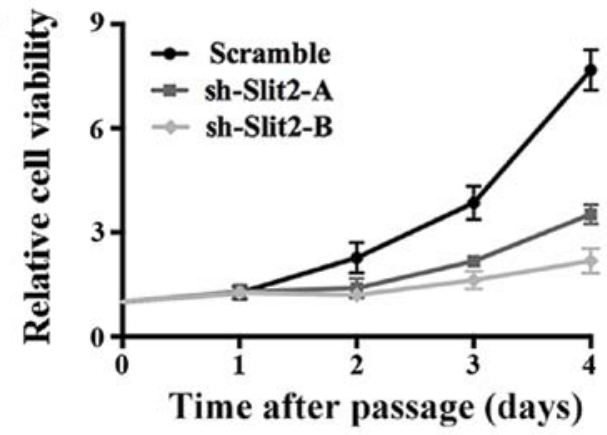

D

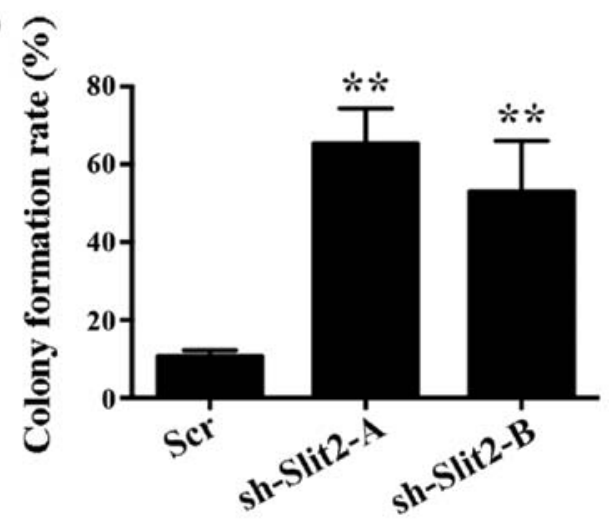

Figure 3. Silencing Slit2 enhances cell viability of K1 cells. (A) Two shRNA constructs effectively decreased Slit2 level in K1 cells. (B) Silencing K1 promoted cell viability reflected by CCK-8 assay. (C and D) Decreased Slit2 expression enhanced clone formation capacity of K1 cells.

Table I. Baseline characteristics in a consecutive series of 196 papillary thyroid cancers.

\begin{tabular}{lc}
\hline Variables & Total $(\%)$ \\
\hline Clinical factor & \\
Gender & \\
Male & $65(33.2)$ \\
Female & $131(66.8)$ \\
Age & $45.7 \pm 12.7(14-80)$ \\
Pathological factor & \\
Maximal tumor size $(\mathrm{cm})$ & $2.0 \pm 1.4(0.2-9.0)$ \\
Multifocality & $24(12.2)$ \\
ETE & $45(23.3)$ \\
HT & $41(20.9)$ \\
LNM & $109(55.6)$ \\
Outcome & $19(9.7)$ \\
Regional recurrence & $2(1.0)$ \\
Distant metastasis & \\
\hline
\end{tabular}

Data are presented as $\mathrm{n}(\%)$ or mean \pm standard deviation (range). ETE, extrathyroidal extension; HT, Hashimoto's thyroiditis; LNM, lymph node metastasis.

Silencing Slit2 enhances cell viability of $K 1$ cells. In order to examine the role of Slit 2 in thyroid cancer proliferation in vitro, we used shRNA mediated silencing of Slit2 in K1 cells. Two shRNAs against Slit2 effectively decreased Slit2
Table II. The associations between Slit2 in tumor tissue according to immunohistochemistry (IHC) and patient outcomes.

\begin{tabular}{|c|c|c|c|c|}
\hline \multirow{2}{*}{$\begin{array}{l}\text { Tumor } \\
\text { tissue }\end{array}$} & \multicolumn{2}{|c|}{$\begin{array}{l}\text { Regional lymph } \\
\text { node metastasis }^{\text {a }}\end{array}$} & \multicolumn{2}{|c|}{$\begin{array}{c}\text { Recurrence } \\
{\text { (local or distant })^{b}}^{\text {local }}\end{array}$} \\
\hline & Negative & Positive & Negative & Positive \\
\hline \multicolumn{5}{|l|}{ Slit2 } \\
\hline- & $20(10.2)$ & $42(21.4)$ & 39 (19.9) & $8(4.1)$ \\
\hline+ & $32(16.3)$ & $40(20.4)$ & $69(34.7)$ & $7(3.6)$ \\
\hline++ & 35 (17.9) & $27(13.8)$ & $70(35.7)$ & $4(2.0)$ \\
\hline
\end{tabular}

expression (Fig. 3A). Then we examined the effect of silencing Slit 2 on cell viability by CCK- 8 assay. A significant increase in cell viability was observed upon Slit2 silencing (Fig. 3B). Moreover, clone formation assay also suggested that silencing Slit2 increased cloning capacity of $\mathrm{K} 1$ cells, indicating that Slit2 is a negative regulator of cell proliferation (Fig. 3C and D).

Slit2 is associated with Warburg effect in K1 cells. It is well accepted that many cancer cells exhibit elevated glucose uptake and lactate production, regardless of oxygen availability, known as aerobic glycolysis or Warburg effect. Enhanced 

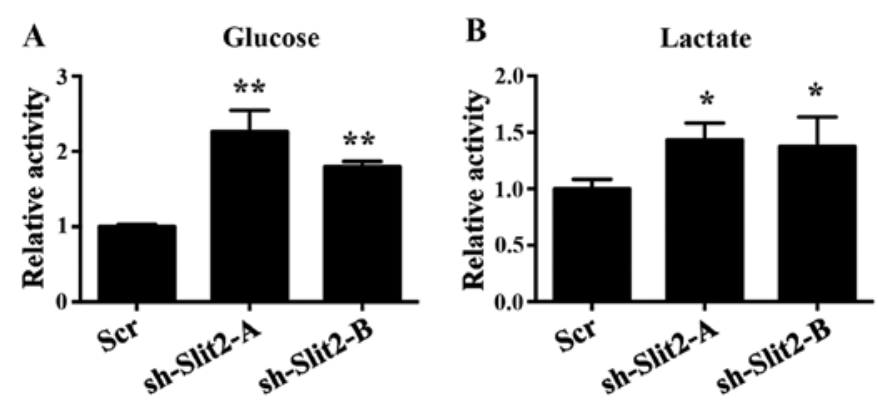

Figure 4. Slit2 is associated with Warburg effect in K1 cells. (A) Glucose uptake was increased significantly in Slit2 knockdown K1 cells. (B) Slit2 has a negative role in lactate production, because silencing Slit2 promoted lactate produced by glycolysis in $\mathrm{K} 1$ cells.
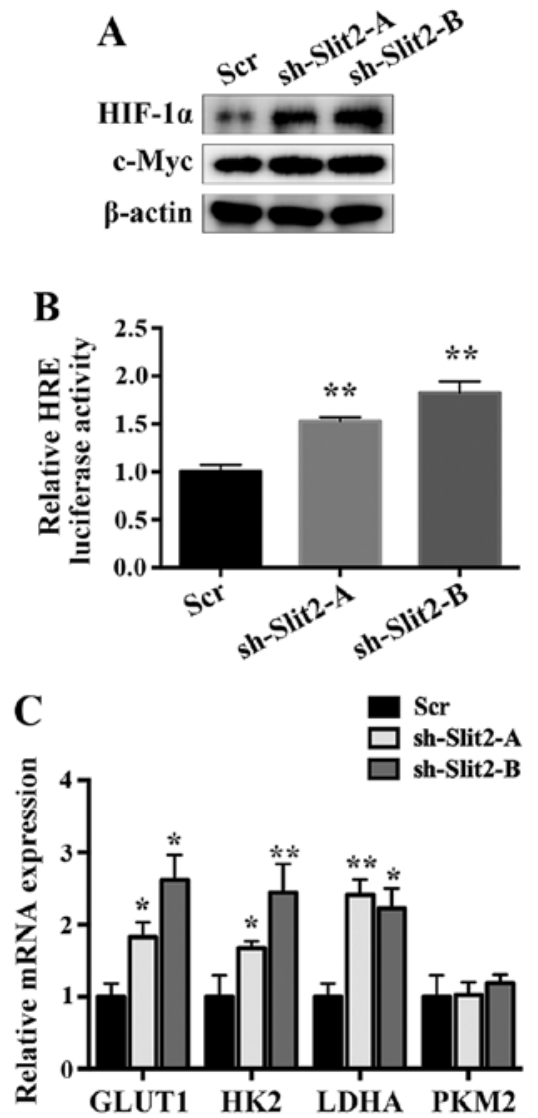

Figure 5. Slit2 regulates HIF $1 \alpha$ and HIF $1 \alpha$ transcriptional activity (A) Slit2 silencing induced upregulation of HIF1 $\alpha$ and c-myc in protein level. (B) HIF1 $\alpha$ transcriptional activity reflected by HRE reporter assay increased in Slit2 knockdown K1 cells. (C) Downregulation of Slit2 increased HIF1 $\alpha$ targeted glycolytic genes including GLUT1, HK2 and LDHA, while PKM2 changed slightly.

glycolysis facilitates uncontrolled proliferation of cancer cells by providing building blocks for macromolecule synthesis and energy source. We next asked whether the effect of Slit2 on cell proliferation was associated with glycolysis. In K1 cells, decreased Slit2 expression enhanced glucose uptake, which is the first step for glucose metabolism (Fig. 4A). Lactate, the key product of the Warburg effect, was not only used as a source for metabolism, but also created an acidic environment that leads to destabilization of extracellular matrix that facilitates
Table III. The negative association between Slit2 and HIF1 $\alpha$ in tumor tissue according to immunohistochemistry (IHC).

\begin{tabular}{lcccc}
\hline & \multicolumn{4}{c}{ HIF1 $\alpha$} \\
\cline { 2 - 5 } $\begin{array}{l}\text { Tumor } \\
\text { tissue }\end{array}$ & - & + & ++ & +++ \\
\hline Slit2 & & & & \\
- & $10(5.1)$ & $30(15.3)$ & $34(17.3)$ & $0(0)$ \\
+ & $4(2.0)$ & $24(12.2)$ & $39(19.9)$ & $8(4.1)$ \\
++ & $7(3.6)$ & $35(17.9)$ & $5(2.6)$ & $0(0)$ \\
\hline
\end{tabular}

Data are presented as $\mathrm{n}(\%)$. Pearson's $\chi^{2}=139.086, \mathrm{P}=0.001$.

metastasis of cancer cells. Our results also demonstrated that decreased Slit2 expression enhanced lactate production of K1 cells (Fig. 4B).

Slit2 regulates HIFl $\alpha$ and HIFla transcriptional activity. $\mathrm{HIF} 1 \alpha$ is a transcription factor that mediated the primary transcriptional response to hypoxic stress of transformed cancer cells. In conjugation with c-myc, they are considered to be key regulators of the Warburg effect. We speculated that Slit2 might regulate glycolysis in part through HIF1 $\alpha$ and c-myc. Consistent with this assumption, we observed an increase in HIF1 $\alpha$ and c-myc protein levels in Slit2 knock-down cells (Fig. 5A). In order to assess the role of Slit2 on HIF1 $\alpha$ transcriptional activity, we performed HRE luciferase reporter assay. Silencing Slit2 increased HRE-luciferase activity, supporting the result that Slit2 regulated HIF1 $\alpha$ protein level (Fig. 5B). As a transcription factor, HIF1 $\alpha$ regulates series of genes in glycolysis process. GLUT1 (glucose transporter 1) is a membrane protein that facilitates glucose transport across the cell membrane, which is subsequently catalyzed to glucose 6-phosphate by HK2 (hexokinase 2). In the last step of glycolysis, L-lactate and NAD are converted to pyruvate and NAHD by LDHA (lactate dehydrogenase A). PKM2 (pyruvate kinase isozyme M2) catalyzes the dephosphorylation of phosphoenolpyruvate to pyruvate, and is responsible for net ATP production. PKM2 is also a HIF1 $\alpha$ target gene. To further confirm the function of Slit2 on HIF1 $\alpha$ transcriptional activity control, we examined the expression of HIFla targeted glycolysis genes upon Slit2 downregulation, and observed that GLUT1, HK2 and LDHA were significantly upregulated. However, the expression of PKM2 was changed slightly, indicating that there may be other mechanisms for PKM2 regulation in thyroid cancer (Fig. 5C).

Slit2 negatively correlates with HIFla expression in PTC samples. Correlation between Slit2 and HIF1 $\alpha$ was analyzed by IHC staining. HIF1 $\alpha$ level was low in Slit2 positive PTC samples (Fig. 6). Statistical analysis demonstrated that there is a negative association between Slit2 and HIF1 $\alpha$ in PTC tissue microarray (Table III).

Schematic representation of the working model. In conclusion, we found that decreased Slit2 expression in PTC sample renders a proliferation advantage to PTC cells, the potential 

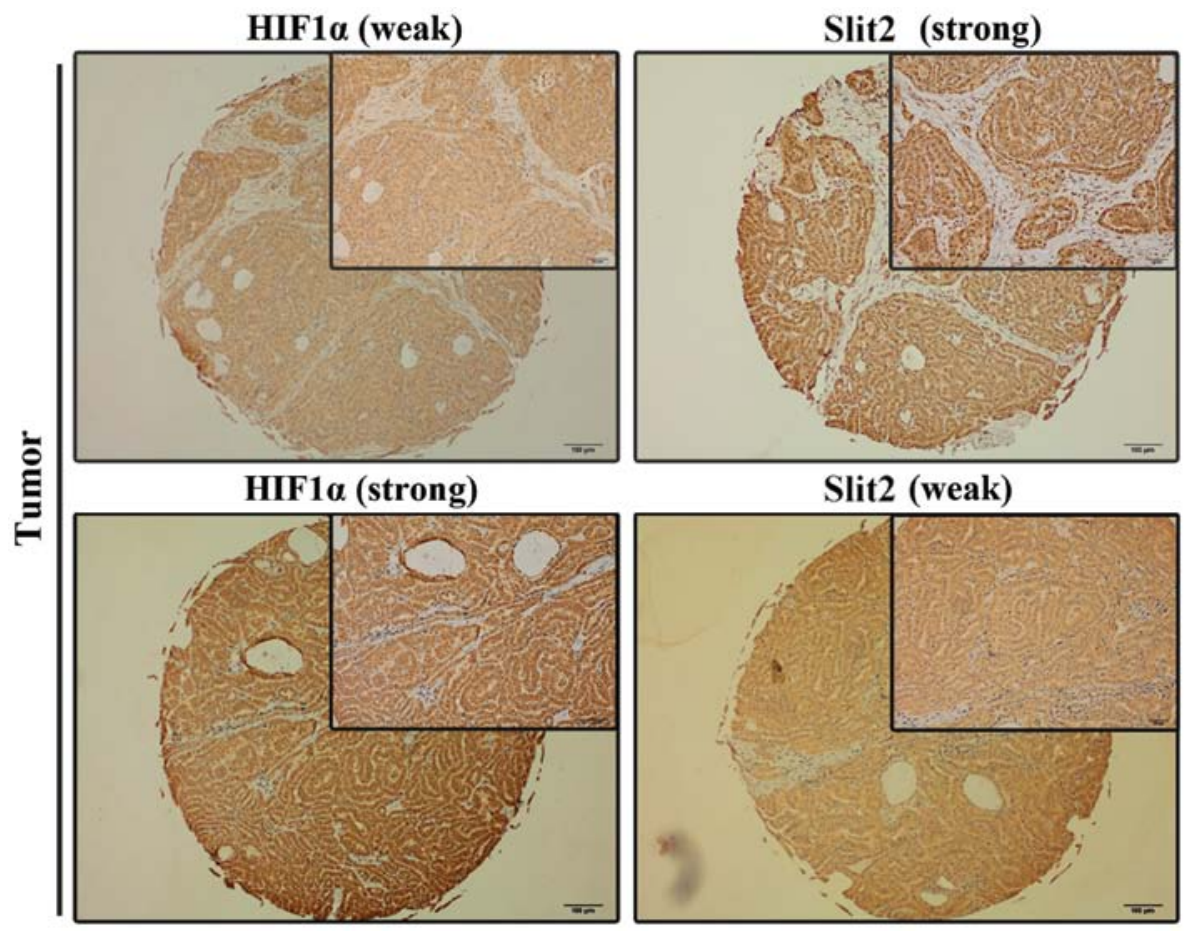

Figure 6. Slit2 negatively correlates with HIF1 $\alpha$ expression in PTC samples. HIF1 $\alpha$ staining was low in Slit2 strongly stained tissue samples. However, HIF1 $\alpha$ exhibited strong staining in Slit2 weakly expressing PTC samples.
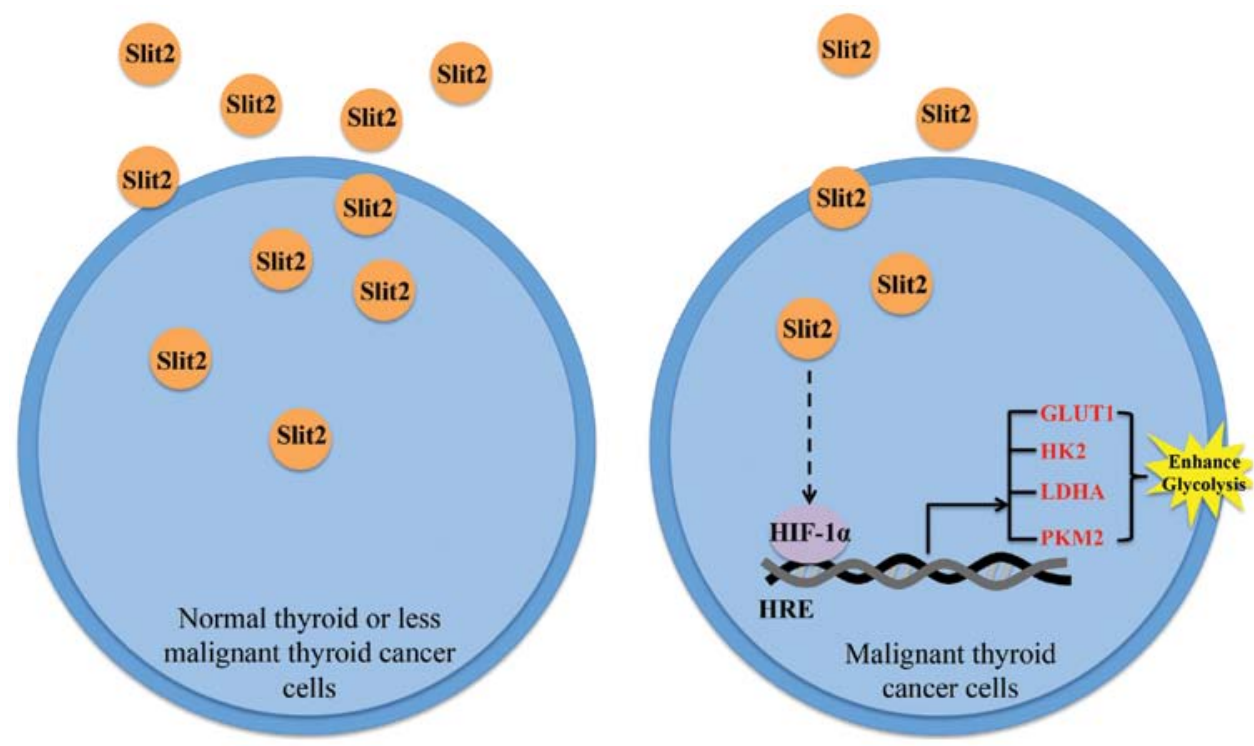

Figure 7. Working model of Slit2 in PTC. Slit2 level was decreased in PTC tumor samples. Decreased Slit2 expression promoted HIF1 $\alpha$ mediated glycolysis.

mechanism may involve the participation of Slit2 in $\mathrm{HIF} \alpha$ control (Fig. 7).

\section{Discussion}

Slit2 plays an important role in the regulation of many signaling pathways such as cell cycle, apoptosis, migration and invasion. In recent years, the role of Slit 2 in cancer has received much attention, though its role in cancer is controversial. Slit2 inactivation by promoter hypermethylation of allele loss in lung, breast, liver, and malignant glioma supported the notion that Slit2 has antitumor function $(17,18)$. Slit2 can also inhibit metastasis of tumor cells through targeting the AKT-GSK3 $\beta$ signaling pathway (19). On the contrary, exogeneous expression of Slit2 induced epithelial-mesenchymal transition (EMT) in colorectal cancer through E-cadherin degradation (20). Moreover, Slit2 could also activate Rho GTPase family proteins such as Racl and Cdc42, which promotes tumor cell migration, thus, making it a positive regulator of metastasis (10). Thus, the role of Slit 2 in cancer is distinct depending on the cancer type. Our previous studies $(15,16)$ in gastric cancer indicated that Slit2 is a tumor suppressor. 
The expression of Slit2 was higher in gastric patients with less advanced clinicopathological features. Mechanistically it regulates cell malignancy through the Akt/ $\beta$-catenin signaling pathway. However, its role in PTC has not been reported.

In the present study, we examined the expression and function of Slit2 in PTC. The mRNA and protein levels of Slit2 in PTC patient samples were examined by qPCR and IHC. Out of 130 paired specimens investigated by qPCR, the expression levels of Slit2 were significant higher in adjacent non-tumor thyroid tissues compared with PTC tissues. Moreover, the expression level of Slit2 was decreased in tumors without LNM. Similarly, IHC analysis data were consistent with mRNA expression results. Using tissue microarray containing samples from 196 patients, we observed that Slit2 protein level decreased in PTC samples and in LNM samples. Taken together, these results indicated that Slit 2 may be a tumor suppressor in PTC. To further validate the role of Slit2 in PTC, we silenced Slit2 expression in the PTC cell line K1. Results indicated that silencing Slit 2 expression promoted cell proliferation. The above results supported the notion that Slit2 is a tumor suppressor in PTC.

To explore the mechanism, we examine the role of Slit2 on PTC cell glucose metabolism transformation. It is well accepted that cellular metabolism influence life and death decisions (21). An emerging theme in cancer biology is that metabolic regulation is tightly and intricately linked to cancer progression, which is due to the basic fact that cell proliferation is tightly regulated by availability of nutrients that provide cells with building blocks for macromolecule synthesis and energy production $(22,23)$. The most characterized is the glucose metabolism transformation (24). PTC is one kind of solid tumors, and sites of the tumor are at a great distance from the supporting blood vessels, thus suffering from hypoxia, acidosis and increased interstitial fluid pressure. To survive under such hypoxic hazardous conditions, cancer cells shift their metabolism pattern, known as hypoxic adaptation to meet demands of sustainable growth and metastasis (25). One such adaptation is glycolysis, also known as Warburg effect, which provides a benefit to the tumors $(26,27)$. Our results demonstrated that silencing Slit2 expression promoted glucose uptake and lactate production in $\mathrm{K} 1$ cells. Increased glucose uptake ensures increased demands for biomass accumulation and ATP requirement (28). Lactate produced by glycolysis is not only used by mitochondrion for further metabolism but also created an acidic microenvironment, which leads to extracellular matrix destabilization that facilitates metastasis $(29,30)$.

The transcription factor HIF1 $\alpha$ is a master regulator of glycolysis and has been implicated in regulating many of the genes that are responsible for the metabolic difference $(31,32)$. Our results have shown that silencing Slit2 increased HIF1 $\alpha$ protein level and transcriptional activity. The transcription of a series of glycolytic rate-limiting enzymes, such as Glut1, HK2 and LDHA, was significantly upregulated. All these results supported the assumption that Slit2 is a negative regulator of glycolysis and HIF1 $\alpha$ is an effector protein.

In conclusion, the present study shows that Slit 2 mRNA and protein levels were significantly reduced in thyroid cancer specimens and were associated with prognosis and disease progression. Moreover, our results provide clinical and in vitro evidence implicating Slit2 functions as a negative regulator in the development and progression of PTC. Further mechanism analysis indicated that Slit2 regulated PTC glycolysis via HIF1 $\alpha$.Thus Slit2 could be used as a prognostic factor and treatment target for PTC.

\section{Acknowledgements}

The present study was supported by funds from the National Science Foundation of China (nos. 81572622 and 81272934 to Q.H.J.) and the Shanghai Rising-Star Program (no. 15QA1401100 to Y.L.W.).

\section{References}

1. Faam B, Ghaffari MA, Ghadiri A and Azizi F: Epigenetic modifications in human thyroid cancer. Biomed Rep 3: 3-8, 2015.

2. Kondo T, Ezzat S and Asa SL: Pathogenetic mechanisms in thyroid follicular-cell neoplasia. Nat Rev Cancer 6: 292-306, 2006.

3. Patel KN and Shaha AR: Poorly differentiated and anaplastic thyroid cancer. Cancer Control 13: 119-128, 2006.

4. Chen AY, Jemal A and Ward EM: Increasing incidence of differentiated thyroid cancer in the United States, 1988-2005. Cancer 115: 3801-3807, 2009.

5. Davies L and Welch HG: Increasing incidence of thyroid cancer in the United States, 1973-2002. JAMA 295: 2164-2167, 2006.

6. Li Y, Nakamura M and Kakudo K: Targeting of the BRAF gene in papillary thyroid carcinoma (Review). Oncol Rep 22: 671-681, 2009.

7. Liu D, Hou J, Hu X, Wang X, Xiao Y, Mou Y and De Leon H: Neuronal chemorepellent Slit2 inhibits vascular smooth muscle cell migration by suppressing small GTPase Rac1 activation. Circ Res 98: 480-489, 2006.

8. Wu W, Wong K, Chen J, Jiang Z, Dupuis S, Wu JY and Rao Y: Directional guidance of neuronal migration in the olfactory system by the protein Slit. Nature 400: 331-336, 1999.

9. Xu Y, Li WL, Fu L, Gu F and Ma YJ: Slit2/Robo1 signaling in glioma migration and invasion. Neurosci Bull 26: 474-478, 2010.

10. Wong K, Ren XR, Huang YZ, Xie Y, Liu G, Saito H, Tang H, Wen L, Brady-Kalnay SM, Mei L, et al: Signal transduction in neuronal migration: Roles of GTPase activating proteins and the small GTPase Cdc42 in the Slit-Robo pathway. Cell 107: 209-221, 2001.

11. Wong K, Park HT, Wu JY and Rao Y: Slit proteins: Molecular guidance cues for cells ranging from neurons to leukocytes. Curr Opin Genet Dev 12: 583-591, 2002.

12. Qiu H, Zhu J, Yu J, Pu H and Dong R: SLIT2 is epigenetically silenced in ovarian cancers and suppresses growth when activated. Asian Pac J Cancer Prev 12: 791-795, 2011.

13. Dallol A, Morton D, Maher ER and Latif F: SLIT2 axon guidance molecule is frequently inactivated in colorectal cancer and suppresses growth of colorectal carcinoma cells. Cancer Res 63: 1054-1058, 2003.

14. Dallol A, Da Silva NF, Viacava P, Minna JD, Bieche I, Maher ER and Latif F: SLIT2, a human homologue of the Drosophila Slit2 gene, has tumor suppressor activity and is frequently inactivated in lung and breast cancers. Cancer Res 62: 5874-5880, 2002.

15. Shi R, Yang Z, Liu W, Liu B, Xu Z and Zhang Z: Knockdown of Slit2 promotes growth and motility in gastric cancer cells via activation of AKT/ $\beta$-catenin. Oncol Rep 31: 812-818, 2014.

16. Shi R, Liu W, Liu B, Xu Z, Chen L and Zhang Z: Slit2 expression and its correlation with subcellular localization of $\beta$-catenin in gastric cancer. Oncol Rep 30: 1883-1889, 2013.

17. Dallol A, Krex D, Hesson L, Eng C, Maher ER and Latif F: Frequent epigenetic inactivation of the SLIT2 gene in gliomas. Oncogene 22: 4611-4616, 2003.

18. Jin J, You H, Yu B, Deng Y, Tang N, Yao G, Shu H, Yang S and Qin W: Epigenetic inactivation of SLIT2 in human hepatocellular carcinomas. Biochem Biophys Res Commun 379: 86-91, 2009. 
19. Chang PH, Hwang-Verslues WW, Chang YC, Chen CC, Hsiao M, Jeng YM, Chang KJ, Lee EY, Shew JY and Lee WH: Activation of Robol signaling of breast cancer cells by Slit 2 from stromal fibroblast restrains tumorigenesis via blocking PI3K/ Akt/ $\beta$-catenin pathway. Cancer Res 72: 4652-4661, 2012.

20. Zhou WJ, Geng ZH, Chi S, Zhang W, Niu XF, Lan SJ, Ma L, Yang X, Wang LJ, Ding YQ, et al: Slit-Robo signaling induces malignant transformation through Hakai-mediated E-cadherin degradation during colorectal epithelial cell carcinogenesis. Cell Res 21: 609-626, 2011.

21. Muñoz-Pinedo C, El Mjiyad N and Ricci JE: Cancer metabolism: Current perspectives and future directions. Cell Death Dis 3: e248, 2012

22. Dang CV: Links between metabolism and cancer. Genes Dev 26: 877-890, 2012

23. Deberardinis RJ, Sayed N, Ditsworth D and Thompson CB: Brick by brick: Metabolism and tumor cell growth. Curr Opin Genet Dev 18: 54-61, 2008.

24. Cairns RA, Harris IS and Mak TW: Regulation of cancer cell metabolism. Nat Rev Cancer 11: 85-95, 2011.

25. Meijer TW, Kaanders JH, Span PN and Bussink J: Targeting hypoxia, HIF-1, and tumor glucose metabolism to improve radiotherapy efficacy. Clin Cancer Res 18: 5585-5594, 2012.
26. Denko NC: Hypoxia, HIF1 and glucose metabolism in the solid tumour. Nat Rev Cancer 8: 705-713, 2008.

27. Keith B, Johnson RS and Simon MC: HIF1 $\alpha$ and HIF2 $\alpha$ : Sibling rivalry in hypoxic tumour growth and progression. Nat Rev Cancer 12: 9-22, 2011.

28. Pereira KM, Chaves FN, Viana TS, Carvalho FS, Costa FW, Alves AP and Sousa FB: Oxygen metabolism in oral cancer: HIF and GLUTs (Review). Oncol Lett 6: 311-316, 2013

29. Bertout JA, Patel SA and Simon MC: The impact of O2 availability on human cancer. Nat Rev Cancer 8: 967-975, 2008.

30. Le A, Cooper CR, Gouw AM, Dinavahi R, Maitra A, Deck LM, Royer RE, Vander Jagt DL, Semenza GL and Dang CV: Inhibition of lactate dehydrogenase A induces oxidative stress and inhibits tumor progression. Proc Natl Acad Sci USA 107: 2037-2042, 2010.

31. Vaupel P, Mayer A and Höckel M: Tumor hypoxia and malignant progression. Methods Enzymol 381: 335-354, 2004.

32. Semenza GL: Defining the role of hypoxia-inducible factor 1 in cancer biology and therapeutics. Oncogene 29: 625-634, 2010. 